\title{
Fuzzy-neural network model for effective control of profitability in a paper recycling plant
}

\author{
Uduak A. Umoh, M.Sc. ${ }^{1}$, Enoch O. Nwachukwu, Ph.D. ${ }^{2}$, Okure U. Obot, Ph.D. ${ }^{1}$, \\ Augustine A. Umoh, Ph.D. ${ }^{3}$ \\ ${ }^{1}$ Department of Computer Science, University of Uyo, Nigeria. \\ ${ }^{2}$ Department of Computer Science, University of Port Harcourt, Nigeria. \\ ${ }^{3}$ Department of Physics, University of Uyo, Nigeria
}

\begin{abstract}
This research develops a fuzzy-neural network model, using Al technologies and applies the model for effective control of profitability in paper recycling to improve production accuracy, reliability, robustness and to maximize profit generated by an industry, despite varying cost of production upon which ultimately profit, in an industry depend. Recycling reduces greenhouse gas emissions, conserves the natural resources on Earth, and saves space in the landfills for future generations of people. A sustainable future requires a high degree of recycling. However, Recycling industries face serious economic problems that increase the cost of recycling. Fuzzy logic has emerged as a tool to deal with uncertain, imprecise, partial truth or qualitative decisionmaking problems, to achieve robustness, tractability, and low cost, but it cannot automatically acquire the rules it uses to make those decisions. Neural networks have the ability to learn, generalize and process large amount of numerical data, but they are not good at explaining how they reach their decisions. The hybrid fuzzy-neural system has the ability to overcome the limitations of individual technique and enhances their strengths to handle financial trading. In order to achieve our objective, a study of a knowledge based system for effective control of profitability in paper recycling is carried out. The Mamdani's Max-Min technique is employed to infer data from the rules developed. This resulted in the establishment of some degrees of influence of input variables on the output. Fuzzy-Neural network model is developed using back propagation and supervised learning methods respectively. The outputs of Fuzzy logic serve as input to the neural network. To reinforce the proposed approach, we apply it to a case study performed on Paper recycling industry in Nigeria. A computer simulation is designed to assist the experimental decision for the best control action. The system is developed using MySQL, NetBeans, Java, MS Excel 2003, MatLab, etc. The obtained simulation and implementation fuzzy-neural results are investigated, compared and discussed.
\end{abstract}

Keywords: Recycling, Fuzzy logic, Neural Network, Profitability, Industrial efficiency

\section{INTRODUCTION}

Urbanization is one of the most evident global changes worldwide. The rapid and constant growth of urban population has led to a dramatic increase in urban solid waste production, with a crucial socioeconomic and environmental impact. However, the growing concern for environmental issues and the need for sustainable development have moved the management of solid waste to the forefront of the public agenda.

Recycling technology has evolved as one of the most useful facilities that help us to maintain an environmentally friendly society. All over the world, the need of recycling is heightened by the increasing awareness of product consumer, on the need to maximize the bundle of benefits from the products brought by them.

As the demand for materials continues to grow and the supply of natural resources continues to dwindle, recycling of materials has become more important in order to ensure sustainability. Recycling reduces greenhouse gas emissions that may lead to global warming. Recycling also conserves the natural resources on Earth, saves space in the landfills for future generations of people. A sustainable future requires a high degree of recycling. However, Recycling industries face serious economic problems 
that increase the cost of recycling (Kumar, 2001), (Kumaran et al, 2001).

The profitability of recycling industries is known to be highly dependent on the effective management of resources and management practices, (Craighill and Powell, 1996) (Cunningham, 1969). Taking the paper recycling industry as a case study, the recycling of paper has contributed in no small measure to the conservation of material and consequent low cost of production.

The ultimate resultant effect of the recycling process is high profit generated due to the low cost of production, hence, the link between profitability and recycling industry. Since the ultimate aim of any capitalist industry is to make profit, the concept of profitability is of great significance in science and engineering (http://www.recyclingtoday.com, http://www.recyclinginternational.com,).

This highlights the need of applying fuzzy-neural network models as one of the best techniques for effective control of profitability in paper recycling production to ensure profit maximization despite varying cost of production upon which ultimately profit, in an industry depend.

Wongthatsanekorn, 2009, developed a goal programming model for plastic recycling system in Thailand. Kufman, 2004, carried out the analysis of technology and infrastructure of paper recycling. Kumar et al, 2008, designed a goal programming model for paper recycling to assist proper management of the paper recycling logistic system, while (Udoakpan, 2002) carried out the financial implication of establishing a paper recycling plant in Nigeria. (Oke et al, 2006) designed a fuzzy logic model to handle the profitability concept in a plastic industry. Oke et al, 2006, developed a Neuro-Fuzzy linguistic approach in optimizing the flow rate of a Plastic Extruder process. Kumar, 2007, designed an optimal blending model for paper manufacturing with competing input materials MisrawatiMisman et al, 2008, studied State-Of-The-Art for Paper Recycling. (Umoh et al, 2010a) developed fuzzy logic model for the control of profitability in paper recycling production. (Umoh, et al, 2010b) designed a fuzzy inference system for profitability control in paper recycling production using Mamdani's approach.

Fuzzy logic is a powerful technique for solving a wide range of industrial control and information processing applications (Akinyokun, 2002). Fuzzy logic controller has its origin with the E. H. Mamdani (Mamdani, 1977 ) researches, based on theories proposed by $L$. Zadeh (Zadeh, 1965). It has emerged as a tool to deal with uncertain, imprecise, partial truth or qualitative decision-making problems to achieve robustness, tractability, and low cost, but it cannot automatically acquire the rules it uses to make those decisions. Neural network has the ability to learn, generalize and process large amount of numerical data and the ability to improve performance over time, but they are not good at explaining how they reach their decision. The hybrid fuzzy-neural system has the ability to overcome the limitations of individual technique and enhances their strengths to handle financial trading.

In order to achieve our objective, a study of a knowledge based system for effective control of profitability in paper recycling is carried out. The Mamdani's Max-Min technique is employed to infer data from the rules developed (Umoh et al, 2010b). This resulted in the establishment of some degrees of influence of input variables on the output. FuzzyNeural network model is developed using back propagation and supervised learning methods respectively. The outputs of Fuzzy logic serve as input to the neural network. To reinforce the proposed approach, we apply it to a case study performed on Paper recycling industry in Nigeria. A computer simulation is designed to assist the experimental decision for the best control action. The system is developed using MySQL Database Management System 6.0, NetBeans 6.5.1, Java 1.6, Microsoft Excel 2003, MatLab Version 7.6.0.342 (R2007b), Microsoft Windows XP version 2002, Pentium IV Personal computer. The obtained simulation and implementation fuzzy-neural results are investigated, compared and discussed.

In section 2 presents the objective while in Section 3 the research methodology is presented. Section 4 presents the model experiment while in Section 5 results of findings are discussed. Finally in Section 6, some recommendations are made and conclusion is drawn.

Research Objective: The objective of this research is to develop a fuzzy-neural network model using Al technologies and apply the model for effective control of profitability in paper recycling to improve production accuracy, reliability, robustness and profit generated by an industry. 


\section{RESEARCH METHODOLOGY}

The Fuzzy-Neural Network Model: Figure 1 shows the architecture of the Fuzzy Neural Network (FNN).
It is basically a five-layer fuzzy rule-based neural network.

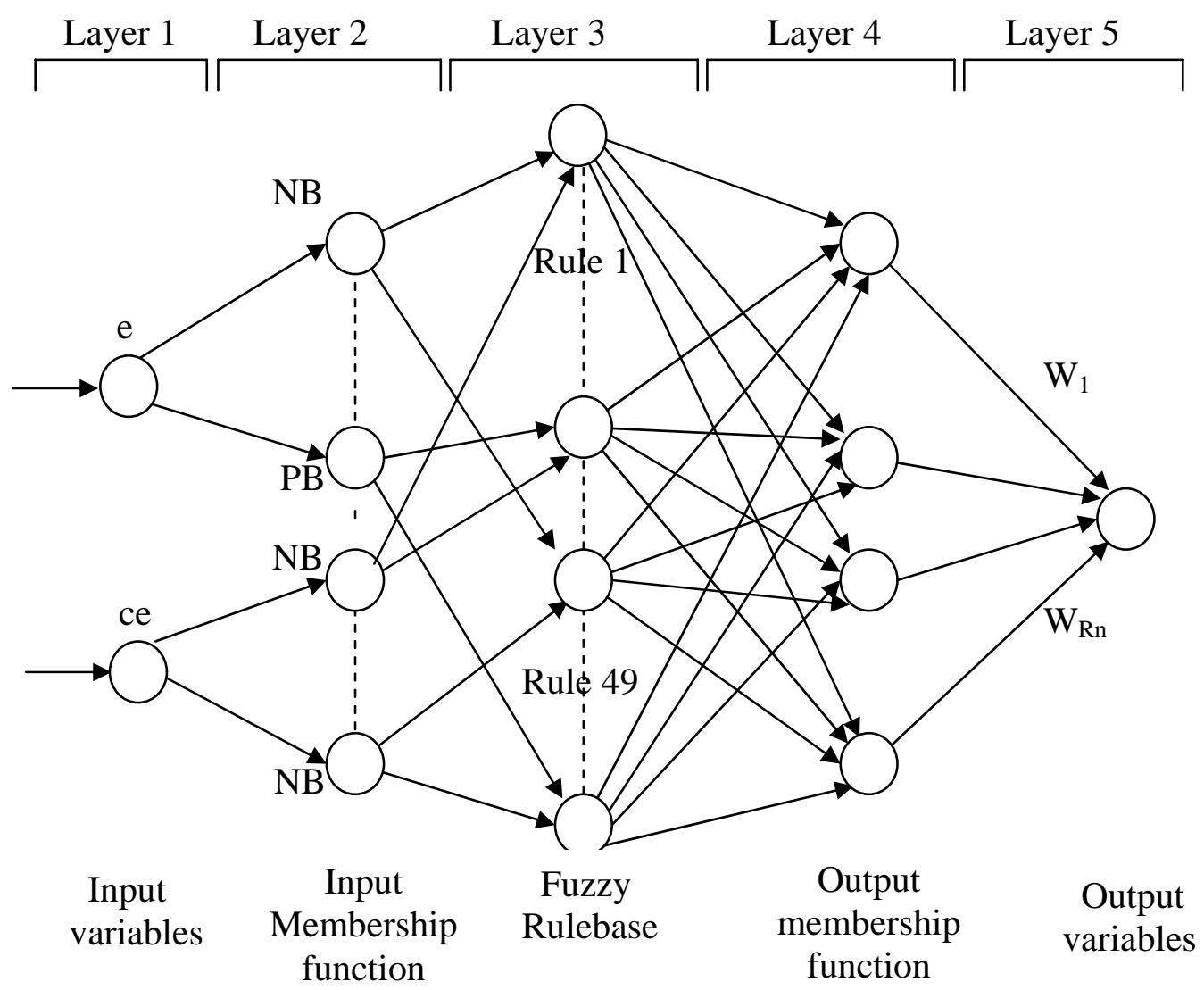

Fig. 1: The Fuzzy-Neural Network Model

Layer 1: $\quad$ The nodes in this layer serves as the fuzzy input linguistic variables, (E, CE) which transmit input values to the next layer directly.

Layer 2: $\quad$ The nodes in this layer are the input membership functions which perform fuzzification of input linguistic variables. They contain 14 neurons corresponding to 14 fuzzy sets of 2 linguistic variables. Seven fuzzy sets of one input linguistic variables are $\{N B, N S, N, Z, P, P S, P B\}$. The membership functions are normal distribution with a range between 0 and 1. Triangular membership functions are proposed in this work to describe the variables in order to suppress noise in the inputs.

$$
\begin{gathered}
\mu_{\mathrm{A}}\left(\mathrm{X}_{\mathrm{i}}: \mathrm{a}_{1}, \mathrm{a}_{2}, \mathrm{a}_{3}\right)=\left[\frac{X_{i}-a_{1}}{a_{2}-a_{1}},\right. \\
\left.\frac{a_{3}-X_{i}}{a_{3}-a_{2}}, 0\right]
\end{gathered}
$$

Where $\mathrm{i}=1,2, \ldots, \mathrm{n}, \mathrm{n}=49$. Where $\mathrm{A}$ can be the term "Negative Big", "Negative Small", Negative", "Zero", "Positive", Positive Small" or "Positive Big". $X_{i}$ is the normalized input, $\left\{a_{1}, a_{2}, a_{3}\right\}$ are the triangular membership function parameters, $a_{1}$ and $a_{3}$ defines the triangular end points while $a_{2}$ defines the triangular peak location. The output of this layer is the degree to which the given input 
satisfies the linguistic label associated to this layer.

Layer 3: Nodes in layer 3 consist of fuzzy rule base; there are 49 neurons in this layer to realize fuzzy inference. The nodes in this layer perform the min-max operation to determine the firing strength of the associated rule. The degrees of truths of the rules are determined for each rule by evaluating the nonzero minimum values using AND operator as shown in (2). They also assign the firing strength to the individual rule output (3), evaluate the individual output value (4) and finally, determine the overall system output by ORing the individual rule output as shown in Equation 5.

net $_{i}^{3}=\alpha_{i}=\min \left(E_{i}\left(x_{0}\right), E_{i}\left(y_{0}\right)\right)$

$C_{i}(w)=a_{i}=n^{3} t_{i}^{3}$

net $_{i}^{3}=C_{i}^{\prime}(w)=\min \left(a_{i 1}, C_{i 1}(w)\right)$

$C_{i j}(w)=\max \left(\right.$ net $_{i 1}{ }^{3}$, net $_{i 2}{ }^{3}$ net $_{i 3}{ }^{3}, \ldots$, net $\left._{i m}{ }^{3}\right)$

where $i=1,2, \ldots n$ and $j 1,2, \ldots, m$

Layer 4: Nodes in this layer represent the membership function outputs

$\operatorname{Net}_{j}^{4}=C_{j}(w) . \Upsilon$

and $\quad f\left(\right.$ net $\left._{j}^{4}\right)=$ net $_{j}^{4}=C_{j}(w) . \Upsilon$

Where $\mathrm{j} 1,2, \ldots ., \mathrm{m}$ and $\Upsilon$ is the link weight

The initial connection weight values are generated randomly or can be assigned directly by an expert. These weights can also be determined outside the network from historical data and then incorporated into the network. The connection weights are subsequently tuned by back propagation algorithm.

Layer 5: This layer performs defuzzification by evaluating the overall system output. This is accomplished by summing up the weights in layer 4 . This is expressed as

net $_{j}^{5} \mathrm{Co(w)}=\sum C_{i}^{\prime}(w) \gamma_{i}$ $f\left(\operatorname{net}^{5}{ }_{C(w)}\right)=\frac{\sum_{i=1}^{n} C_{i}^{\prime}(w) \gamma_{i}}{\sum_{i=i}^{n} \gamma_{i}}=0^{y}$

Weights of this layer are tuned using back propagation algorithm and the error between the desired output $\left(\mathrm{y}^{\mathrm{k}}\right)$ and computed output $\left(\mathrm{O}^{\mathrm{k}}\right)$ are determined.

The Supervised Learning Procedure: The supervised learning method is proposed for this design with the objective of minimizing the error function $E$ as defined in equation 9 by means of $a$ learning algorithm.

$E_{k}=1 / 2\left[y^{k}-O^{k}\right]^{2}$

Where, $y^{k}$ is the desired (expected) output and $o^{k}$ is the actual (computed) output.

In our fuzzy-neural network, we derive our learning algorithm from the back propagation algorithm of (Obot, 2007). This algorithm is studied and modified to suit the requirements for this research paper as stated:

In training our fuzzy-neural network, the output weight is updated and the new output weight is computed and obtained using equation 10.

$W_{k j}(n+1):=w k_{j}(n)+\eta \delta^{\circ}{ }_{k} x_{j}+\omega \sum \Delta w_{j k}(n)$

where $\mathrm{n}$ is the training cycle (epochs).

$w_{k}(n)$ is the weight between the output and hidden neurons at epoch $(n)$

$w_{k}(n+1)$ is the weight between the output and hidden neurons at epoch $(n+1)$

$\eta$ is the Learning rate factor of 0.7

$\delta^{\circ}{ }_{k}$ is the output error signal, called the local gradient. $x_{j}$ is the input to neuron $j$

$\omega$ is the momentum factor

$\Sigma \Delta \mathrm{w}_{\mathrm{jk}}$ is the cumulative weights change of the output neuron.

The learning process is iterated until an acceptable minimum error between desired output $y^{k}$ and computed output $o^{k}$ is achieved.

\section{RESULTS AND DISCUSSION}

Our Fuzzy-Neural network system was developed using MatLab Version 7.6.0.342 (R2007b), MySQL Database Management System 6.0, NetBeans 6.5.1, 
Java 1.6, Microsoft Excel 2003, Microsoft Windows XP version 2002, Pentium IV Personal computer. An application example of FNN using the developed system is presented. The example was the control of profitability in paper recycling production for profit maximization. In this application, three input parameters, which are thought to have an influence on profit of the industry $(\boldsymbol{y})$, was selected. They include cost price of production of recycled quantity $(x 1)$, selling price of recycled quantity $(x 2)$, quantity recycled $(x 3)$. These parameters constitute the fuzzy logic input variables used to generate the fuzzy logic model. Error and change in error terms of the model was defined and evaluated and membership functions constructed for each variable. A total of 400 sample data sets are obtained through fuzzy inference system developed (Umoh et al, 2010b.). The outputs of fuzzy inference engine form the input to the neural networks. These data sets was obtained and used for training the network.

The number of output membership function nodes was set for each input variable with maximum of 6 and minimum or 2 , after studying the distribution patterns of the data samples. Figure 2 shows the leaning converging curve. After the fuzzy-neural networks $\mathrm{n}$ was trained, test data was fed to the trained network to predict the profitability of paper recycled production. Figure 3 shows the graph of the Fuzzy-Neural Networks results versus the Fuzzy logic results.

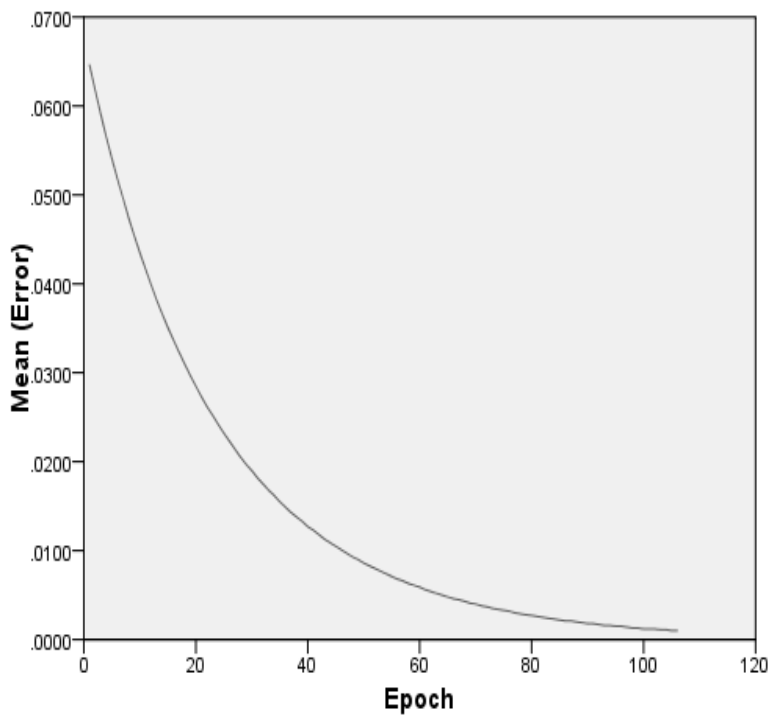

Fig. 2: Error versus Epoch showing A Learning Convergence Curve for the Network of Profitability Training
The error measures based on Mean Square Error (MSE) and Correlation Coefficient (R) was performed with 400 datasets. MSE was computed to measure the average of the squares of the difference between fuzzy neural results and the desired output with Mean Square Error value (MSE) of 0.001, and the difference between the fuzzy logic result and the desired output with MSE value of 0.022 . It was defined by

$$
M S E=\frac{\sum_{i=1}^{n}\left(y_{i}-O_{i}\right)^{2}}{N}
$$

Where $\mathrm{y}=$ desired output, $\mathrm{O}=$ network computed output; $\mathrm{N}=$ number of dataset.

Correlation coefficient (R) was computed to measures the degree of fit between Fuzzy-Neural Networks estimated values and desired values of output target with $R$ value of 0.95 ; this indicated a perfect linear relationship. The difference between the desired output values and fuzzy logic output values are correlated with $R$ value of 0.91 . While the correlation between the fuzzy logic output and fuzzyneural output gives 0.87 .

It was defined by the formula:

$$
R=\frac{\sum(X-\bar{X})(Y-\bar{Y})}{\sqrt{\sum(X-\bar{X})^{2} \sum(Y-\bar{Y})^{2}}}
$$

Where, $Y=$ desired $\mathrm{Y},{ }^{\wedge} \mathrm{X}=$ estimated $\mathrm{Y}$, and $\vec{Y}, \vec{X}=$ the means of $Y$. Its limits are $0 \leq R \geq 1$. $Y=$ Profitability level. The correlation coefficient ranges from +1 to -1 . +1 indicates a perfect positive linear relationship between $X$ and $Y$ that is when $X$ increases, $y$ also increases by the same quantity, and -1 indicates a perfectly negative linear relationship between $x$ and $y$ showing that when $x$ increases, $y$ decreases by the same quantity. On the other hand, when $R$ equals 0 , the model does not explain any of the variation in $\mathrm{Y}$. The closer $\mathrm{R}$ is to 1 , the better the fit of the model (Obot, 2008).

Figure 3 and Table 1 compare the performance of FNN with that of Fuzzy Logic model where, $Y$ (Desired Output) is the Desired Profitability Throughput, $Y$ (FNN Output) is the Predicted Profitability Throughput by FNN and Y(FL Output) is 
the Predicted Profitability outcome by Fuzzy logic Model.

Table 1: Comparison of the performance between Fuzzy-Neural Networks and Multiple Regression Model

\begin{tabular}{|c|c|c|c|}
\hline Dataset & \begin{tabular}{|l|} 
Y(Desired \\
Output)
\end{tabular} & \begin{tabular}{|l|l} 
Y(FNN \\
Output)
\end{tabular} & $\begin{array}{l}\text { Y(FL } \\
\text { Output) }\end{array}$ \\
\hline 1 & 0.6500 & 0.6459 & 0.5000 \\
\hline 2 & 0.8000 & 0.7943 & 0.6500 \\
\hline 3 & 1.0000 & 0.9679 & 0.9000 \\
\hline 4 & 0.9000 & 0.8912 & 0.7500 \\
\hline 5 & 0.6500 & 0.6460 & 0.5000 \\
\hline 6 & 0.9000 & 0.8908 & 0.7500 \\
\hline 7 & 0.8500 & 0.8436 & 1.0000 \\
\hline 8 & 0.8500 & 0.8428 & 0.7000 \\
\hline 9 & 0.6500 & 0.6457 & 0.5000 \\
\hline 10 & 0.9000 & 0.8907 & 0.7500 \\
\hline 11 & 0.8500 & 0.8436 & 1.0000 \\
\hline 12 & 0.9500 & 0.9338 & 0.8000 \\
\hline 13 & 0.6500 & 0.6792 & 0.5000 \\
\hline 14 & 0.8000 & 0.7953 & 0.6500 \\
\hline 15 & 0.8500 & 0.8434 & 1.0000 \\
\hline 16 & 0.9000 & 0.8904 & 0.7500 \\
\hline 17 & 0.6500 & 0.6468 & 0.5000 \\
\hline 18 & 0.8000 & 0.7944 & 0.6500 \\
\hline 19 & 0.9500 & 0.9345 & 0.8000 \\
\hline 20 & 0.8500 & 0.8438 & 1.0000 \\
\hline 21 & 0.6500 & 0.7773 & 0.5000 \\
\hline 22 & 0.6500 & 0.6704 & 0.5000 \\
\hline 23 & 0.6500 & 0.6462 & 0.5000 \\
\hline 24 & 0.6500 & 0.7211 & 0.5000 \\
\hline 25 & 0.6500 & 0.7503 & 0.5000 \\
\hline 26 & 0.6500 & 0.7595 & 0.5000 \\
\hline 27 & 0.6500 & 0.6457 & 0.5000 \\
\hline 28 & 0.6500 & 0.6792 & 0.5000 \\
\hline 29 & 0.6500 & 0.7109 & 0.5000 \\
\hline 30 & 0.6500 & 0.6464 & 0.5000 \\
\hline$\ldots \ldots \ldots \ldots$ & $\ldots \ldots \ldots \ldots$ & $\ldots \ldots \ldots \ldots$ & $\ldots \ldots \ldots \ldots$ \\
\hline 400 & 0.8500 & 0.8436 & 1.0000 \\
\hline \multirow[t]{2}{*}{ Measures } & MSE & 0.001288 & 0.022219 \\
\hline & $\mathbf{R}$ & 0.958815 & 0.913463 \\
\hline
\end{tabular}

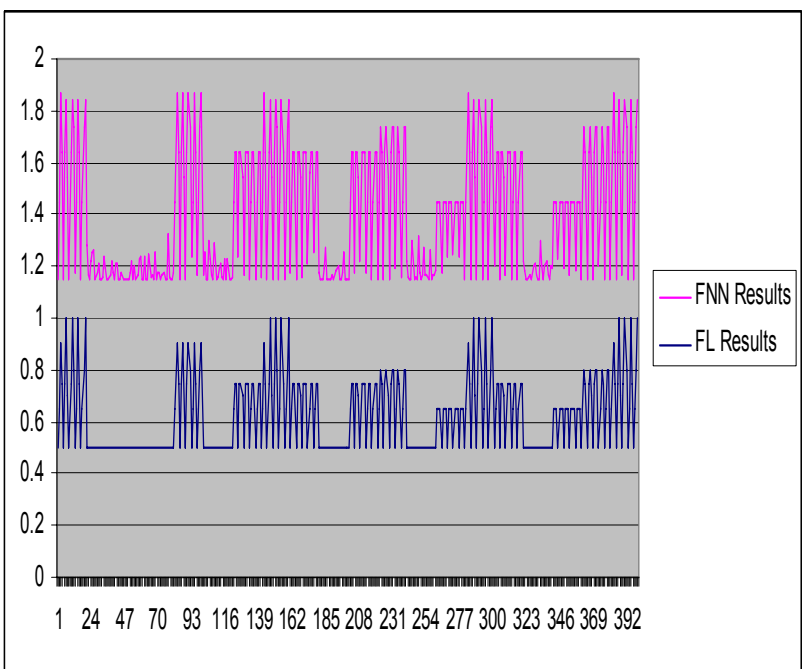

Fig. 3: The Graph of Fuzzy Logic versus Fuzzy-Neural Network Results for Profitability Control.

The results showed that FNN performs much better than the fuzzy logic model in terms of MSE and correlation $(R)$ values. The superior performance of FNN may be attributed to the effectiveness of the fuzzy-neural network architecture.

The result of the application agrees with general observation that the integration of fuzzy logic and neural network gives a more accurate performance, while fuzzy logic model is less accurate in cases where the predicting trend is irregular as is the case in this study.

\section{CONCLUSION}

A fuzzy neural network (FNN) model for effective control of profitability is developed. The FNN, which is basically five-layer fuzzy rule-based neural network architecture, is used to control profitability in paper recycling production and its performance is compared with fuzzy logic model. The results show that the fuzzy-neural networks perform better and are superior to fuzzy logic models in terms of control accuracy.

The fuzzy-neural network system developed in this project is a toolkit for profitability control design. It brings trade-off analysis into the model process, allowing industrialists to adopt a "control" approach. By bringing multi-domain analysis to the industrialist's desktop, profitability control model and development methodology can optimize model performance, eliminates iterations, and shortens time to production and maximize production profit. This dramatically reduces the cost of production, and accelerates production operations. 
The fuzzy logic neural network hybrid system proposed in this paper thus provides a better solution to the problems of profitability control than fuzzy logic and conventional models.

\section{REFERENCES}

Akinyokun, o. c. (2002). Neuro- Fuzzy Expert System for Evaluation of Human Resources Performance. First Bank of Nigeria PLC Endowment Fund Lecture Series 1, Delivered at the Federal University of Technology, Akure, December, 10, 2002.

Craighill, A.L. and Powell J.C. 1996. "Lifecycle Assessment and Economic Evaluation of Recycling: A Case Study". Resource, Conservation \& Recycling. 17(2):75-96.

Cunningham, M.T. 1969. "The Application of Product Life Cycles to Corporate Strategy: Some Research Findings". European Journal of Marketing. 3(1):32-44.

Fuzzy Logic Control Tool Box, User's Guide Version 7.5.0.342 (R2007). August 15, 2007, the Maths Works Inc.

Jadarat, M. A. K., and Al-Nimr, (2009), Fuzzy Logic Controller Deployed for Indoor Air Quality Control in Naturally Ventilated Environments. Journal of Electrical Engineering, Vol 60, No. 1, 12-17.

Kumar, R. (2007), Optimal Blending Model for Paper Manufacturing With Competing

Input Materials Pati, POMS 18th Annual Conference, Dallas, Texas, U.S.A.

Kumaran, D.S., Ong. S.K., Tan, R.B.H. and Nee, A.Y.C. (2001). "Environmental lifecycle cost analysis of products", Environmental Management and Health, 12(3), 260-276.

Mamdani, E.H. (1977), Application of fuzzy logic to approximate reasoning using linguistic systems. Fuzzy Sets and Systems 26, 1182-1191.
Misrawati M., Sharifah R. W. A., and Zainuddin A. M. (2008). State-Of-The-Art for Paper Recycling. International Conference on Science and Technology (ICSTIE), Universiti Teknologi MARA, Pulau Pinang, Malaysia,

Obot, O. U. (2008). Fuzzy rule-base frame work for the management of tropical diseases. Int. Journal of Medical Engineering and Informarics, 1(1): 7 - 17.

Oke, S. A., Johnson, A. O., Charles-Owaba,O. E., Oyawale, F. A. and Popoola, I. O. (2006a). Application of Fuzzy Logic Concept to Profitability Quantification in Plastic Recycling. Pacific Journal of Science and Technology. 7(2),163-175.

Oke, S. A., Johnson, A. O., Charles-Owaba,O. E., Oyawale, F. A. and Popoola.I. O. (2006b). A NeuroFuzzy Linguistic Approach in Optimizing the Flow Rate of a Plastic Extruder Process. International Journal of Science \& Technology 1(2), 115-123.

Umoh, U.A., Nwachukwu, E. O., and Obot, O.U.. 2010a. Fuzzy Rule-based Framework for Effective Control of Profitability in a Paper Recycling Plant. Global Journal of Computer Science and Technology, (10)10: 56-67

Umoh, U.A., E.O. Nwachukwu, and O.U. Obot. 2010b. Fuzzy Inference System for Effective Control of Profitability in a Paper Recycling Plant: Mamdani's Design Approach. Pacific Journal of Science and Technology, 10(2):391-407.

Wongthatsanekorn, W. (2009) A Goal Programming Approach for Plastic Recycling System in Thailand. Proceedings of World Academy of Science, Engineering and Technology Vol. 37, ISSN 2070-3740

Zadeh, L. A. (1965), Fuzzy Sets, Information and Control, 1965

http://www.recyclingtoday.com http://www.recyclinginternational.com 